\title{
Clonidine versus butorphanol as adjuvant to $0.5 \%$ hyperbaric bupivacaine to enhance the onset and duration of subarachnoid blockade with postoperative analgesia during orthopaedic surgeries-A randomized study
}

\author{
Kumkum Gupta ${ }^{1}$, Manish Jain ${ }^{1}$, Prashant K Gupta ${ }^{2}$, Shikha Agarwal ${ }^{1}$, K S Bhatia ${ }^{1}$, VP Singh ${ }^{1}$, Bhawana Rastogi ${ }^{*}$ and Meetu Singh $^{1}$ \\ ${ }^{1}$ Department of Anaesthesiology and Critical care in NSCB Subharti Medical College, Meerut, UP, India \\ ${ }^{2}$ Department of Radio-diagnosis and interventional imaging in NSCB Subharti Medical College, Meerut, UP, India
}

\begin{abstract}
Background: Intrathecal adjuvants to local anaesthetic enhance the quality and duration of spinal anaesthesia. The present study was aimed to comparatively evaluate the clonidine and butorphanol as adjuvants to intrathecal hyperbaric bupivacaine for subarachnoid blockade during orthopaedic surgeries.

Patients and method: Sixty four adult consented patients of both gender of ASA physical status I and II scheduled for elective orthopaedic surgeries under subarachnoid blockade, were analyzed. All enrolled patients were divided into two groups of 32 patients each to receive either $3.5 \mathrm{ml}$ of $0.5 \%$ hyperbaric bupivacaine with $0.2 \mathrm{ml}$ of clonidine $30 \mu \mathrm{g}$ (Group I) or with $0.2 \mathrm{ml}$ of butorphanol $0.20 \mathrm{mg}$ (Group II). Patients were compared for onset and duration of sensory and motor blockade, intraoperative hemodynamic changes, time to first rescue analgesic and side effects by using Analysis of variance (ANOVA), Mann Whitney U test and chi-square test.

Result: The demographic profile of patients was comparable. The onset of sensory block to T10 (3.4 \pm 1.7 versus $3.91 \pm 2.2$ min) and time to achieve complete motor block $(11.23 \pm 4.7$ versus $12.9 \pm 3.2 \mathrm{~min})$ was significantly earlier in patients of clonidine Group. The time to first request for analgesia was $278.7 \pm 29.6$ min in clonidine group and $218.6 \pm 21.9 \mathrm{~min}$ in patients of butorphanol group. The incidences of bradycardia were more in patients of clonidine group with statistically significant difference while incidences of hypotension, respiratory depression, shivering or any other side effects were negligible.
\end{abstract}

Conclusion: Clonidine is a useful adjuvant to intrathecal hyperbaric bupivacaine during orthopaedic surgeries and can be considered as alternative to butorphanol.

\section{Introduction}

Neuraxial anesthesia is commonly performedfor all surgical procedures carried on lower abdomen, pelvis and lower limbs to provide adequate surgical anesthesia and analgesia. It has a definite advantage over general anesthesia by blocking nociceptive impulses from the operative site, reduced blood loss and decreased incidence of deep vein thrombosis. Spinal anesthesia is unparalleled in the way that a small mass of drug, virtually devoid of systemic pharmacologic effect, can produce surgical anesthesia but hemodynamic instability due to sympathetic blockade impedes the effective and safe use of spinal anesthesia. These effects are proportional to the level of sympathetic blockade [1].

Anti-nociceptive synergism between local anesthetic and intrathecal adjuvants, opioids or a 2 agonist, allow the reduction in the dosages of both drugs, reduce the unwanted side effects attributable to both drugs and improves the perioperative analgesia [2-4].

Clonidine, a selective partial $\alpha-2$ adrenergic agonist is potent analgesic and free from opioid-related side effects of pruritus, nausea and vomiting, respiratory depression and urinary retention. It is known to increase both sensory and motor blockade of local anesthetics [5]. Butorphanol is a synthetic lipophilic opioid analgesic and used intravenously in balanced anesthetic technique but there are very few studies available in the literature on the clinical characteristics of intrathecal butorphanol [6-8]. Therefore we planned the present study to compare the efficacy and safety of intrathecal clonidine with butorphanol as adjuvants to $0.5 \%$ hyperbaric bupivacaine in patients scheduled for orthopaedic surgeries.

\section{Patients and methods}

After approval by the Institutional Ethical Committee and written informed consent, 64 patients of ASA (American Society of Anaesthesiologist) physical status I and II, aged 21 to 58 years of both genders, scheduled for various orthopaedic surgeries under subarachnoid blockade, were enrolled for this prospective double blind randomized study. All patients were subjected to pre-anaesthetic assessment prior to enrolment and patients with history of diabetes mellitus, cardiac or pulmonary disease, hypertension, spinal deformity,

Correspondence to: Bhawana Rastogi, Department of Anaesthesiology and Critical Care in NSCB Subharti Medical College, Meerut (UP), India, Tel: +918449114984; Fax: +91-0121-2439056; E-mail: dr.bhawana@gmail.com

Key words: bupivacaine, butorphanol, clonidine, orthopaedic surgery, subarachnoid blockade

Received: April 05, 2015; Accepted: May 07, 2015; Published: May 10, 2015 
Gupta K (2015) Clonidine versus butorphanol as adjuvant to 0.5\% hyperbaric bupivacaine to enhance the onset and duration of subarachnoid blockade with postoperative analgesia during orthopaedic surgeries-A randomized study

severe liver disease or impaired renal functions, skin infection at site of injection, coagulation disorders, allergy to local anaesthetic, history of opioid dependence or neurological disorders and patient's refusal to technique were excluded from the study. Before enrolment for the study, patients were properly explained on the method of sensory and motor assessments.

Patients were randomly divided in a double-blind manner into two equal groups of 32 patients each according to computer generated random number table. They received study solution of $3.5 \mathrm{ml}$ of $0.5 \%$ hyperbaric bupivacaine either with $0.2 \mathrm{ml}$ of clonidine $30 \mu \mathrm{g}$, (Group I) or $0.2 \mathrm{ml}$ of butorphanol $0.20 \mathrm{mg}$, (Group II). The drug was prepared by an anaesthesiologist who was blinded to study protocol and was not involved in further assessment of patient. They were admitted prior to surgery and their 6 hours fasting was ensured.

After arrival of patient into operation theatre, routine monitoring of non- invasive blood pressure (NIBP), heart rate (HR), electrocardiogram (ECG) and finger pulse oximetry (SpO2) was commenced and they were preloaded with crystalloid solution at rate of $10 \mathrm{ml} \mathrm{kg}^{-1}$ before initiation of subarachnoid blockade. Under all aseptic condition, lumbar puncture was performed at L3-4 inter-vertebral disc space with a $25 \mathrm{G}$ Quincke's spinal needle in the sitting position by midline approach. After identification of correct placement by free flow of cerebral spinal fluid, $3.7 \mathrm{ml}$ of study solution was injected at rate of $0.2 \mathrm{ml} /$ seconds and then the patient was immediately turned to supine position.

The sensory block was assessed by bilateral pin prick method along the mid-clavicular line using a short bevelled 26-G hypodermic needle at 2 minutes interval till the surgical anaesthesia was achieved. The onset of sensory blockade was defined as the time from intrathecal injection to the occurrence of sensory block at dermatome level T10. The maximal cephalic level of sensory blockade and the time taken to attain, was also noted.

The onset of motor blockade of the lower extremities was evaluated by observing toes movements and modified Bromage scale (0-3), if possible, at every two minutes. Definition of motor blockade according to the modified Bromage scale [9] is, 0- able to flex the whole lower limb at the hip (full motor activity), 1- able to flex the knee but unable to raise the leg at the hip, 2- able to planter flex the ankle but unable to flex the knee, 3- no movement of lower limb (no toes movements).The onset of motor block was defined as the time from intrathecal injection to the absence of toes activity. The surgical anesthesia was considered effective when T10 dermatome was anesthetized.

Postoperatively the sensory and motor block levels were assessed at 15 min intervals until normal sensations returned. Duration of analgesia was taken from onset of spinal anesthesia to time of administration of first rescue analgesic, assessed using a 0-10 linear visual analogue scale (VAS) every 15 min. Rescue analgesia was given with inj. Diclofenac sodium $75 \mathrm{mg}$ intramuscularly when (VAS $>4$ ).

Intraoperative hemodynamic parameters of heart rate and blood pressure were recorded at every $5 \mathrm{~min}$ in the first hour and thereafter every $10 \mathrm{~min}$ until the patient was transferred to the post anesthesia room. For the present study, hypotension was defined as a fall in systolic blood pressure of more than $25 \%$ of base line or less than $100 \mathrm{~mm} \mathrm{Hg}$ and was treated with additional crystalloid solution and incremental dosages of mephenteramine $6 \mathrm{mg}$. Bradycardia (heart rate $<56$ beats/ min) was treated with intravenous atropine. Supplemental oxygen was administered throughout the orthopaedic surgery. Side effects of pruritus, nausea, vomiting, respiratory depression (respiratory rate $<10$ breaths/min or peripheral oxygen saturation $<90 \%$ ) on air or shivering was observed and managed symptomatically.

The results obtained at the end of study, are tabulated as Mean \pm SD and analysed using Stat Graphics Centurion (version16.2). The demographic data for categorical variables was compared using chi-square test and statistical significance in mean difference was done by using analysis of variance (ANOVA). The spinal blockade characteristics were compared using Mann Whitney U test. A p value of $<0.05$ was considered to indicate statistical significance.

\section{Results}

The present study was successfully conducted on 64 enrolled patient of comparable demographic profile. There was no protocol deviation and all were included for data analysis. Patients were cooperative with subsequent assessment of subarachnoid blockade characteristics (Table-1).

The sensory and motor blockade characteristics of both groups are shown in Table 2. The mean onset time of sensory analgesia at $\mathrm{T} 10$ dermatome was $3.4 \pm 1.7 \mathrm{~min}$ in patients of clonidine group and 3.9 $\pm 2.2 \mathrm{~min}$ in patients of butorphanol group. The mean time to reach highest level (T 6) for sensory block showed no statistically significant difference. The mean time taken for motor block up to Bromage scale 3 was $11.23 \pm 4.7 \mathrm{~min}$ in patients of clonidine group while it took 12.9 $\pm 3.2 \mathrm{~min}$ in patients of butorphanol group but showed no statistical difference. The duration of sensory analgesia was $218.6 \pm 21.9 \mathrm{~min}$ in patients of butorphanol group as compared to patients of clonidine group $(278.7 \pm 29.6 \mathrm{~min})$ with statistical significant difference. The need for rescue analgesia with Diclofenac sodium (VAS $>4$ ) was significantly earlier in patients of butorphanol group.

The hemodynamic characteristics of base line preoperative heart rate and systolic blood pressure along with intraoperative hemodynamic variations of heart rate and systolic blood pressure at different time intervals are shown in Table 3. All patients showed a decrease in heart rate and mean blood pressure from the baseline values but the

Table 1. Demographic profile of patients $N=64$.

\begin{tabular}{|l|l|l|}
\hline Parameters & Group I & Group II \\
\hline Age (years) & $56.6 \pm 2.8$ & $54.9 \pm 4.2$ \\
\hline Weight $(\mathrm{kg})$ & $62.56 \pm 8.37$ & $64.36 \pm 7.54$ \\
\hline Gender M:F & $23: 9$ & $21: 11$ \\
\hline ASA Grade I/II & $19 / 13$ & $21 / 11$ \\
\hline Duration of surgery & $109.68 \pm 23.74$ & $108.89 \pm 26.22$ \\
\hline
\end{tabular}

Table 2. Sensory and motor blockade profile.

\begin{tabular}{|l|l|l|l|}
\hline Parameters & Group I & Group II & P value \\
\hline $\begin{array}{l}\text { Onset time of Sensory block at T } 10 \\
\text { level (min) }\end{array}$ & $3.4 \pm 1.7$ & $3.91 \pm 2.2$ & 0.083 \\
\hline Median cephalic sensory level & T6 (4-8) & T6 (4-7) & 0.076 \\
\hline $\begin{array}{l}\text { Time taken to achieve sensory blockade } \\
\text { at most cephalic level (min) }\end{array}$ & $7.4 \pm 2.7$ & $9.13 \pm 3.8$ & 0.069 \\
\hline $\begin{array}{l}\text { Time taken to achieve complete motor } \\
\text { block (min) }\end{array}$ & $11.23 \pm 4.7$ & $12.9 \pm 3.2$ & 0.37 \\
\hline $\begin{array}{l}\text { Time taken for total regression of } \\
\text { sensory block at S1(min) }\end{array}$ & $316.7 \pm 29.6$ & $278.6 \pm 23.88$ & $0.001^{* *}$ \\
\hline \begin{tabular}{l} 
Duration of motor block (min) \\
\hline $\begin{array}{l}\text { Time to administer first rescue } \\
\text { analgesia (min) }\end{array}$
\end{tabular} & $310.63 \pm 26.05$ & $283.26 \pm 39.63$ & $0.035^{*}$ \\
\hline
\end{tabular}

*P value is significant, $* *$ P value is highly significant 
Gupta K (2015) Clonidine versus butorphanol as adjuvant to 0.5\% hyperbaric bupivacaine to enhance the onset and duration of subarachnoid blockade with postoperative analgesia during orthopaedic surgeries-A randomized study

Table 3. Hemodynamic parameters of heart rate and systolic blood pressure.

\begin{tabular}{|c|c|c|c|c|}
\hline Parameters & Heart rate (beats/min) & & SBP ( mm Hg) & \\
\hline Groups & Group I & Group II & Group I & Group II \\
\hline Preoperative & $97.3 \pm 8.16$ & $104.5 \pm 3.95$ & $135.2 \pm 1.92$ & $129.5 \pm 3.97$ \\
\hline $5 \mathrm{~min}$ after $\mathrm{SA}$ & $71.7 \pm 6.34$ & $95.3 \pm 4.78$ & $118.5 \pm 2.72$ & $117.2 \pm 4.78$ \\
\hline $10 \mathrm{~min}$ & $70.8 \pm 7.21$ & $94.5 \pm 2.43$ & $115.6 \pm 3.71$ & $117.3 \pm 2.75$ \\
\hline $15 \mathrm{~min}$ & $65.4 \pm 4.28$ & $95.2 \pm 3.45$ & $110.4 \pm 2.64$ & $114.4 \pm 4.70$ \\
\hline $20 \min$ & $61.5 \pm 3.45$ & $87.6 \pm 1.98$ & $112.3 \pm 1.68$ & $112.7 \pm 3.76$ \\
\hline $25 \mathrm{~min}$ & $63.2 \pm 4.67$ & $82.4 \pm 1.76$ & $106.8 \pm 1.60$ & $111.9 \pm 2.74$ \\
\hline $30 \mathrm{~min}$ & $59.8 \pm 2.38$ & $79.7 \pm 2.57$ & $103.7 \pm 3.60$ & $107.3 \pm 4.73$ \\
\hline $45 \mathrm{~min}$ & $63.1 \pm 3.47$ & $77.2 \pm 5.21$ & $103.3 \pm 4.61$ & $109.2 \pm 5.67$ \\
\hline $60 \mathrm{~min}$ & $60.3 \pm 7.61$ & $79.6 \pm 3.89$ & $108.9 \pm 2.93$ & $108.4 \pm 6.62$ \\
\hline $75 \mathrm{~min}$ & $61.4 \pm 6.36$ & $72.4 \pm 6.38$ & $104.4 \pm 1.65$ & $110.7 \pm 3.68$ \\
\hline $90 \mathrm{~min}$ & $58.7 \pm 4.93$ & $71.3 \pm 4.78$ & $106.5 \pm 1.76$ & $118.1 \pm 8.72$ \\
\hline Postoperative & $62.9 \pm 5.69$ & $74.9 \pm 7.18$ & $109.3 \pm 2.67$ & $112.5 \pm 9.75$ \\
\hline
\end{tabular}

SA: Spinal Anesthesia, SBP: Systolic Blood Pressure, ${ }^{*} \mathrm{p}$ value: $<0.05$ significant

magnitude of decrease was lesser with patients of butorphanol group as compared to patients of clonidine group. No incidence of hypotension $(\mathrm{SBP}<100 \mathrm{~mm} \mathrm{Hg})$ occurred in any patient hence the vasopressor was not used. The incidence of bradycardia (heart rate $<60$ beats/min) was higher in patients of clonidine group with statistically significant difference and was managed with intravenous atropine $(0.25-0.5 \mathrm{mg})$.

No significant variations in respiratory rate or desaturation were observed in any patients during the study period. The ventilatory frequency and peripheral oxygen saturation were comparable between the groups though patients of clonidine were well sedated as compared to patients of butorphanol group. Side effects of pruritus, shivering, nausea and vomiting did not occur. There were no transient neurological symptoms in any patients.

\section{Discussion}

Neuraxial blockade with local anesthetic agents reduces the dynamic pain and surgical stress response by preventing the central sensitization to pain. The local anesthetic agents have a limited duration of action, systemic toxicity and lead to perioperative hemodynamic instability which threatens the effective and safe use of spinal anesthesia in orthopaedic patients. Different anesthetic techniques are employed to lower the incidence of the hemodynamic complications by using either the low dose of local anesthetic agents or addition of adjuvants to local anesthetic [1].

Although low dose bupivacaine reduces the cardiovascular effects but it is not enough to provide an adequate level of sensory blockade. If an analgesic drug like opioid or a 2 agonist is added to hyperbaric bupivacaine, it would reduce the required dose of bupivacaine and will also ensure the adequate surgical analgesia, as they exert their action independently via different mechanisms. The intrathecal adjuvants are synergistic with local anesthetic agents to intensify the sensory block without increasing the level of sympathetic block [10].

The combination of local anaesthetic and adjuvants effectively inhibit multiple areas of neuronal excitability to provide a dose sparing effects of local anaesthetics. The analgesic effect of clonidine is mediated spinally through activation of post synaptic $\alpha-2$ receptors in substantia gelatinosa of the spinal cord to enhance the sensory and motor blocks of local anaesthetics without increasing the incidence of side effects of respiratory depression [11]. Butorphanol exert its action by opening the $\mathrm{K}^{+}$channels and reducing the $\mathrm{Ca}^{++}$influx, resulting in inhibition of transmitter release to enhance the analgesia and sedation without detrimental hemodynamic effects [12]. Bupivacaine acts mainly by blockade of voltage-gated $\mathrm{Na}^{+}$channels in the axonal membranes and presynaptic inhibition of calcium channels.

A combination of these effects may explain the observed synergism between bupivacaine and clonidine/ butorphanol in the present study. The synergism was characterized by enhanced somatic analgesia without affecting the cephalic spread of bupivacaine. We observed that maximal cephalic sensory level was comparable between the groups. The mean time to two-segment regression, regression to L3 dermatome and time to first rescue analgesic request was significantly more in clonidine group than butorphanol group. Our findings were similar to study conducted by Van Tuijl et al. $[13,14]$ and Dobrydnjov et al. [15] suggesting that the dose of intrathecal clonidine does not affect the mean maximal level of sensory block.

Intrathecal clonidine when combined with local anesthetic significantly potentiates the intensity and duration of motor blockade possibly due to the fact that $\alpha-2$ adrenoreceptors agonists induce cellular modification in the ventral horn of the spinal cord to facilitate the action of local anesthetic and prolongation in sensory block can be due to vasoconstrictive effect of clonidine [16,17]. In our study we also observed enhanced intensity and duration of motor block in patients of clonidine group as compared to butorphanol group with statistically significant difference.

The principal observation of the present study was that the addition of $30 \mu \mathrm{g}$ of clonidine or $0.20 \mathrm{mg}$ of butorphanol to intrathecal $0.5 \%$ hyperbaric bupivacaine intensified the sensory blockade and increased the duration of analgesia without increasing the duration of motor blockade. The onset of sensory blockade was enhanced in patients of clonidine group as compared to butorphanol group. These observations are in consistence with the results of Vinita et al. [18]

Both these adjuvants to bupivacaine did not cause any significant increase in motor blockade, butorphanol combination with hyperbaric bupivacaine causes less intense motor blockade than clonidine combination. This is in consistent with previous studies.

A significant fall was observed in the arterial blood pressure after intrathecal clonidine administration in our study. The fall in blood pressure occurred at 20-35 minutes after spinal anesthesia. Clonidine produces sympatholysis and reduces arterial blood pressure but no episodes of hypotension ( $\mathrm{SBP}<100 \mathrm{~mm}$ of $\mathrm{Hg}$ ) were recorded in any patient. The heart rate decreased after subarachnoid blockade in 
Gupta K (2015) Clonidine versus butorphanol as adjuvant to 0.5\% hyperbaric bupivacaine to enhance the onset and duration of subarachnoid blockade with postoperative analgesia during orthopaedic surgeries-A randomized study

patients of both groups but more incidences of bradycardia needing intravenous atropine occurred in patients of clonidine group with statistically significant difference between both groups. These observations are similar to the observations of Dobrydnjov et al. [15].

Dose dependent sedation is another central effect of $\alpha-2$ adrenergic agonist which occurred after their administration via intravenous, epidural or intrathecal routes. Sedation did not occur in patients of the present study because we have used intrathecal clonidine in low dose. All patients were comfortable and awake. Niemi et al. [19] and Acalovschi et al. [20] observed significant sedation in patients receiving clonidine. They might have administered the clonidine in higher dosages. Their observation does not coincide with present study.

Saxena et al. evaluated the safest and effective lowest dose of intrathecal clonidine as adjuvant to hyperbaric bupivacaine and concluded that addition of clonidine in dose of $30 \mu \mathrm{g}$ would reduce the onset time with increase in the duration of spinal anesthesia [21]. In the present study, we used clonidine in dose of $30 \mu \mathrm{g}$ as adjuvant to bupivacaine and our results are consistent with their results.

BS Sethi et al. studied the efficacy of analgesic effects of intrathecal clonidine as adjuvant to bupivacaine and concluded that clonidine significantly increases the duration of spinal analgesia with clinically insignificant influence on hemodynamic parameters and level of sedation [22]. In present study, postoperative analgesia was better and prolonged in patients receiving intrathecal clonidine as compared to intrathecal butorphanol.

We observed in the present study that intrathecal clonidine and butorphanol as adjuvant to $0.5 \%$ hyperbaric bupivacaine lead to adequate surgical analgesia with stable intraoperative hemodynamic effects. No episodes of nausea and vomiting, headache, shivering and urinary retention were observed in any patients during the study.

\section{Conclusion}

Intrathecal clonidine is useful adjuvant to $0.5 \%$ hyperbaric bupivacaine for orthopaedic surgeries due to early onset and enhanced duration of surgical anesthesia, no respiratory depression and prolonged analgesia. No side effects related to clonidine or butorphanol occurred during the study period. Clonidine as adjuvant to intrathecal bupivacaine can be considered as alternative to opioids for orthopaedic surgeries.

\section{References}

1. Churchill Davidson HC (2003) Spinal and epidural block. In: Wylie \& Churchill Davidson -A practice of Anesthesia, ( $7^{\text {th }}$ Edn.), London: 608 .

2. Milne B, Cervenko FW, Jhamandas K, Sutak M (1985) Intrathecal clonidine: analgesia and effect on opiate withdrawal in the rat. Anesthesiology 62: 34-38. [Crossref]

3. Gabriel JS, Corden V (2001) Alpha-2 agonists in regional anesthesia and analgesia. Curr Opin Anaesthesiol 14: 271-277. [Crossref]

4. Julião MC, Lauretti GR (2000) Low-dose intrathecal clonidine combined with sufentanil as analgesic drugs in abdominal gynecological surgery. J Clin Anesth 12 : 357-362. [Crossref]

5. Brian D Sites, Micheal Beach, Russel Biggs (2003) Intrathecal Clonidine Added to a Bupivacaine-Morphine Spinal Anesthetic Improves Postoperative Analgesia for Total Knee Arthroplasty. AnesthAnalg 2003; 96: 1083-8. [Crossref]

6. Zeedick John F (1977) Butorphanol- a new, potent, parenteral analgesic. Current Therapeutic Reasearch 21: 802-808.

7. Kaur M, Katyal S, Kathuria S, Singh P (2011) A comparative evaluation of intrathecal bupivacaine alone, sufentanil or butorphanol in combination with bupivacaine for endoscopic urological surgery. Saudi J Anaesth 5: 202-207. [Crossref]
8. Kumar B, Williams A, Liddle D, Verghese M (2011) Comparison of intrathecal bupivacaine-fentanyl and bupivacaine-butorphanol mixtures for lower limb orthopedic procedures. Anesth Essays Res 5: 190-195. [Crossref]

9. Bromage PR (1978) Epidural Analgesia. Philadelphia: WB Saunders, pp: 144.

10. Agarwal D, Chopra M, Mohta M, Sethi AK (2014) Clonidine as an adjuvant to hyperbaric bupivacaine for spinal anesthesia in elderly patients undergoing lower limb orthopedic surgeries. Saudi J Anaesth 8: 209-214. [Crossref]

11. Elia N, Culebras X, Mazza C, Schiffer E, Tramèr MR (2008) Clonidine as an adjuvant to intrathecal local anesthetics for surgery: systematic review of randomized trials. Reg Anesth Pain Med 33: 159-167. [Crossref]

12. Vangipuram RC, Anuja Arvind Goyal, Vikas Singh (2013) A study of addition of Inj. Butorphanol to hyperbaric Inj. Bupivacaine given intrathecally to patients undergoing lower segment caesarean section: A randomized, controlled trial. Medical J Dr. D. Y. Patil University 6: 156-160.

13. Van Tuijl, WA, van Klei, DBM, van der werff (2006) The effect of addition of intrathecal clonidine to hyperbaric bupivacaine on postoperative pain and morphine requirements after Caesarean section: a randomized controlled trial. Br J Anaesth 97 365-370. [Crossref]

14. van Tuijl I, Giezeman MJ, Braithwaite SA, Hennis PJ, Kalkman CJ, et al. (2008) Intrathecal low-dose hyperbaric bupivacaine-clonidine combination in outpatient knee arthroscopy: a randomized controlled trial. Acta Anaesthesiol Scand 52: 343-349. [Crossref]

15. Dobrydnjov I, Axelsson K, Thörn SE, Matthiesen P, Klockhoff H, et al. (2003) Clonidine combined with small-dose bupivacaine during spinal anesthesia for inguinal herniorrhaphy: a randomized double-blinded study. Anesth Analg 96: 1496-1503. [Crossref]

16. Jamliya DRH, Vansola DR, Shah DBJ, Chauhan DL (2012) Effect of Clonidine Addition to Hyperbaric $0.5 \%$ Bupivacaine For Spinal Anaesthesia In Lower Limb Surgery [A Comparative Study]. NJIRM. 3: 113-119.

17. Thakur A, Bhardwaj M, Kaur K, Dureja J, Hooda S, et al. (2013) Intratheca clonidine as an adjuvant to hyperbaric bupivacaine in patients undergoing inguinal herniorrhaphy: A randomized double-blinded study. J Anaesthesiol Clin Pharmacol 29: 66-70. [Crossref]

18. Singh Veenita, Gupta LK, Singh GP (2006) Comparison among intrathecal fentanyl and butorphanol in combination with bupivacaine for lower limb surgeries. $J$ AnesthClinPharmacol 22: 371-375.

19. Niemi L (1994) Effects of intrathecal clonidine on duration of bupivacaine spinal anaesthesia, haemodynamics, and postoperative analgesia in patients undergoing knee arthroscopy. Acta Anaesthesiol Scand 38: 724-728. [Crossref]

20. Acalovschi I, Bodolea C, Manoiu C (1997) Spinal anesthesia with meperidine. Effects of added alpha-adrenergic agonists: epinephrine versus clonidine. Anesth Analg 84: 1333-1339. [Crossref]

21. Saxena H, Singh S, Ghildiyal S (2010) Low Dose Intrathecal Clonidine with Bupivacaine improves onset and duration of block with hemodynamic Stability. Internet Journal of Anesthesiology 23: 1.

22. BS Sethi, Mary Samuel, Deepak Sreevastava (2007) Efficacy of Analgesic Effects of Low Dose Intrathecal Clonidine as Adjuvant to Bupivacaine. Indian J Anaesth 51: 415-419.

Copyright: (C2015 Sar1 S. This is an open-access article distributed under the terms of the Creative Commons Attribution License, which permits unrestricted use, distribution, and reproduction in any medium, provided the original author and source are credited. 